\title{
Consensus of the Society of Hair Testing on hair testing for chronic excessive alcohol consumption 2011
}

\begin{abstract}
1. Alcohol is a legal compound in many countries and is consumed in much higher amounts in comparison to other drugs of abuse and by a much higher portion of the population. Compared to other substances, the detection of chronic excessive alcohol consumption by hair analysis has some specific characteristics.
\end{abstract}

2. Currently, according to the World Health Organization and a literature survey, chronic excessive alcohol drinking corresponds to an average consumption of $60 \mathrm{~g}$ of pure ethanol per day over several months.

3. For clinical and forensic purposes, there is a demand to establish chronic excessive alcohol consumption.

4. The direct determination of ethanol itself in hair is not possible due to its volatility and its potential absorption from external sources. Instead, the minor ethanol metabolites ethyl glucuronide (EtG) and/or fatty acid ethyl esters (FAEE) should be measured in hair as direct alcohol consumption markers.

5. After absorption, a small fraction of ethanol is conjugated with glucuronic acid during phase II metabolism to form EtG.

6. EtG is a polar water-soluble substance, stable but sensitive to cosmetic treatment and whose incorporation is not biased by natural hair color.

7. Either gas or liquid chromatography coupled to (tandem) mass spectrometry with deuterated EtG as internal standard should be used to test for EtG in hair.

8. The cut-off for EtG in hair to strongly suggest chronic excessive alcohol consumption is proposed at $30 \mathrm{pg} / \mathrm{mg}$ scalp hair measured in the $0-3$ up to $0-6 \mathrm{~cm}$ proximal segment. If samples less than $3 \mathrm{~cm}$ are used the results should be interpreted with caution.

9. FAEE are formed after alcohol consumption by different enzymes in blood and human tissues.

10. FAEE are insoluble in water and stable at neutral $\mathrm{pH}$ but are sensitive to hair treatment at alkaline $\mathrm{pH}$.
11. The following four esters, ethyl myristate, ethyl palmitate, ethyl oleate and ethyl stearate should be quantified. For interpretation, the sum of the concentrations of these four esters should be used.

12. Headspace solid phase microextraction in combination with gas chromatography-mass spectrometry and use of deuterated FAEE's as internal standards is a suitable technique for determination of FAEE in hair.

13. The cut-off for the sum of the four esters in hair to strongly suggest chronic excessive alcohol consumption is proposed at $0.5 \mathrm{ng} / \mathrm{mg}$ scalp hair measured in the $0-3 \mathrm{~cm}$ proximal segment. If the proximal $0-6 \mathrm{~cm}$ segment is used the proposed cutoff is $1.0 \mathrm{ng} / \mathrm{mg}$ scalp hair. If samples less than $3 \mathrm{~cm}$ are used the results should be interpreted with caution.

14. FAEE are sensitive to cosmetic treatment but their incorporation is not biased by natural hair color.

15. Either EtG or FAEE can be used independently for chronic excessive alcohol consumption assessment. For mutual confirmation and for exclusion of false positive or false negative results the determination of both parameters can be useful.

16. Proficiency tests for determination of EtG and FAEE are recommended in order to enable equal standards of analysis and interpretation in different laboratories.

17. This consensus applies to the determination of chronic excessive alcohol consumption only. This consensus is not applicable for determination of abstinence from alcohol or moderate consumption of alcohol.

18. It is not advisable to use the results of the hair testing for alcohol markers in isolation.

19. This statement was adopted on June 16th 2009 by the Society of Hair Testing during the meeting in Roma (Italy) and then revised on March 22nd 2011 during the meeting in Chamonix (France) and will be reviewed within the next two years. 